\title{
Incidence and Predictors of Systemic Hypertension among Cohort of HIV/AIDS Infected Patients in Health Facilities of South Ethiopia
}

\author{
Alemu Tamiso Debiso \\ School of Public Health, College of Medicine and Health Sciences, Hawassa University, Hawassa, Ethiopia \\ Email: tamisodebiso@gmail.com
}

How to cite this paper: Debiso, A.T. (2020) Incidence and Predictors of Systemic Hypertension among Cohort of HIV/AIDS Infected Patients in Health Facilities of South Ethiopia. Advances in Infectious Diseases, 10, 47-63.

https://doi.org/10.4236/aid.2020.102005

Received: February 1, 2020

Accepted: April 20, 2020

Published: April 23, 2020

Copyright $\odot 2020$ by author(s) and Scientific Research Publishing Inc. This work is licensed under the Creative Commons Attribution International License (CC BY 4.0).

http://creativecommons.org/licenses/by/4.0/

\section{(c) (i) Open Access}

\begin{abstract}
Growing body of epidemiologic evidence suggests that there are a number of modifiable risk factors that lead to increased incidence of hypertension in low income countries. Besides non modifiable factors like age, highly active antiretroviral treatments are believed to be increase the risk of hypertension in Human Immuno Virus patients. But studies disagree as to whether there are a greater incidence of hypertension among HIV/AIDS patients and the role of antiretroviral therapy. The Study was conducted in public health institution of Gamo-Gofa zone by using Retrospective cohort study design. Simple random sampling techniques with stratified sampling with proportional to size allocation were used to select HIV patients. The data were collected by registered Anti Retroviral Treatment Provider nurses, by using structured questionnaire which was adapted from the World Health Organization STEPS instrument. The data were cleaned to check for its completeness, consistency and the presence of missed values and variables, and then it was entered into a pre-designed format in Epi-Info version 7 and transferred to SPSS version 21 and Stata for further analysis. Frequencies, mean, median, quartile and standard deviation were used to describe the data. After necessary assumption of logistic regression model was checked, bivariable and multiple logistic regression models were fitted to see the predictors of hypertension. A total of $834 \mathrm{HIV} /$ AIDS infected (95.4\% response rate) were included in this study. The mean systolic and diastolic BP were $115.7 \mathrm{mmHg}( \pm 16.1 \mathrm{SD})$ and 74.9 $\mathrm{mmHg}( \pm 12.4 \mathrm{SD})$. The cumulative incidence of hypertension was $20 \%$ (95\% CI: 19.9 - 20.03), in equal proportion in Pre-HAART (20.1\%) and HAART (20.0\%) receiving patients. The study revealed modifiable; monthly income (AOR; 2.26, 95\% CI $(1.26,4.05)$ ), Occupational status (AOR, 0.36, 95\% CI;
\end{abstract}


$(0.17,0.78)$ ), Body mass index (AOR: 12.6, (95\% CI: 5.32, 29.8)) and nonmodifiable family history of hypertension (AOR: $2.18,95 \% \mathrm{CI}: 1.32,3.58$ ) as important predictors of hypertension among HIV infected patients. There was high incidence of hypertension among HIV infected patients which may reflect insights for intervention. The investigators accept the null hypothesis that there is association between HAART intervention and incidence of Hypertension.

\section{Keywords}

HAART, HIV/AIDS, Incidence, Predictors, Systemic Hypertension, Ethiopia

\section{Introduction}

Despite little due attention; hypertension is an important public health problem accounting for 1 billion people illness, $6 \%$ of worldwide death and 7.1 million deaths peranum [1] [2]. It is responsible for $62 \%$ of cerebro-vascular disease and $49 \%$ ischemic heart disease [3]. The incidence of hypertension in general increase with age, higher in urban than rural area, in obesity than thin, with high dietary salt intake than low and low dietary intake of calcium than high calcium intake [1] [2] [3], this is not only risk factors for hypertension so far and some study also emphasis association of HAART (highly active antiretroviral therapy) with prevalence's of hypertension among HIV infected people [4].

Since its introduction of HAART can decrease morbidity and mortality rates among HIV-infected patients [5]. Besides this, Potent antiretroviral therapy (HAART) has resulted in lasting suppression of HIV replication, reduction of opportunistic infections and malignancies associated with AIDS, and have had a substantial impact on the survival rate and quality of life of infected individuals [6].

However, the potential to keep these patients under treatment for decades may be limited by a variety of metabolic and cardio-vascular abnormalities observed in patients on HAART, including dyslipidaemia, fat redistribution, insulin resistance, hypertension [4] [7], and coronary ischemia [8].

In the period prior to HAART, high blood pressure in infected patients was often associated with complications related to HIV, such as renal failure and vasculopathy [9] and in post-HAART period, some studies have raised the possibility that HAART may also induce Hypertension [10] [11] [12], through the acceleration of atherogenesis and subsequent hardening of the vessel wall [13].

Though the possibility mentioned above, studies on blood pressure in HIV infected patients show conflicting results regarding the increased prevalence of disease among patients on HAART and the role of different treatment regimens in its genesis [7] [14] [15] [16].

Given the importance of the getting the burden and risk factors for Hypertension and the urgency in adopting preventive measures for cardiovascular dam- 
age secondary to systemic hypertension among patients with HIV/AIDS on antiretroviral treatment, this study will assess the incidences and predictors of systemic hypertension among cohort of HIV patients at health institution of Gamo-Gofa Zone and try to reduce death toll and to improve quality of life of people infected by HIV AIDS.

\section{Materials and Method}

\subsection{Study Area}

The Study was conducted in health institution of Gamo-Gofa zone which is located about $505 \mathrm{~km}$ south west from Addis Ababa. A zone has a population of $1,960,417$ and of this 960,604 was males and 999,813 were females. It has 15 districts and two town administration. With this there are three hospitals and 73 health centres (including upgrading health centres) offering health care services for the population. All three hospitals and 14 health centre offer HIV chronic care services There are 8933 of people living with HIV/AIDS who have follow up at health institution of the Gamo-Gofa zone, among them 3985 are on HAART and 4948 are on Pre ART.

\subsection{Study Design}

Retrospective cohort study design was implemented from January 2015 to May 2016.

\subsection{Study Population}

All HIV infected adult patients' age $\geq 18$, who has followed up at health institution of Gamo Gofa zone and HIV infected adult patients age $\geq 18$, who has follow up at selected health institution of Gamo Gofa zone were source and study population respectively. Furthermore All HIV patients on HAART and PreHAART, who have completed charts was included in the study.

\subsection{Sample Size and Sampling Procedure}

Sample size was calculated for first specific objective by using proportion of hypertension from Addis Ababa (31.5\%) and Gondar hospital (28.3\%), considering Z-score corresponding to $95 \% \mathrm{CI}$, 5\% degree of precision, design effect of two and ten percent non response rate; it gives 728 and 686 respectively. The sample size was determined for Predictors also by using StatCalc program of Epi-Info version 7 with considering the following assumptions; confidence level of $95 \%$, power of $80 \%$, exposed to non-exposed ratio $3: 1$, design effect of 2 , fifteen percent lost to follow up, Odds ratio and its proportion obtained from previous Literature.

Based on the sample size calculated above, sample size for triglyceride was 380 which was large, so considering design effect and lost to follow up, 874 (582 ART and 292 patients on Pre-ART) will be selected (Table 1).

Two hospitals (Sawula and Arba Minch hospital) and four health center (Sa- 
wula health, Birbir, and Arba Minch health center) was selected by simple random sampling. Proportional to size allocation of sample was distributed to health institution. After random allocation, randomly generated number was used to pick out card number of an individual on Pre-ART and HAART, and then all information was accessed from the card and patients through measurements and face to face interview (Table 2).

\subsection{Study Variables}

1) Dependent variables: Incidence of systemic hypertension

\section{2) Independent variables}

Socio-economic and demographic variables (Ethnicity, Income, education, household indicators, occupation, age, sex, and residences), Life style and behavioral variables (tobacco and alcohol use, physical inactivity, intake of fruit and vegetables), Biochemical test variables (Measured weight, height, waist, Timed walk, and pedometer) and Medical history and HIV related variables (Family history of HPN, stage of RVI, Duration on HAART, type of treatment received, family history of DM, history of UTI) were used to collect and analyze data.

\subsection{Data Collection Instruments and Measurements}

The data was collected by ART nurses, who were trained; in the respective health institution after consent from each respondent is obtained through interviewbased, pre-coded and pretested structured questionnaire which was adapted from the WHO STEPS instrument [17]. This instrument was used to collect data

Table 1. Table showing Sample size calculated based on selected factors, 2016.

\begin{tabular}{ccccccccc}
\hline Variables & CI & Power & Unex: Ex & Disease/Ex & OR & Ex & N-Ex & Total \\
\hline Sex (male/female) & - & - & $3 / 1$ & 63.67 & 1.86 & 132 & 44 & 176 \\
Duration on HAART (5+) & - & - & - & 40.23 & 2.62 & 105 & 35 & 105 \\
Triglycerides (150+) & - & - & - & 59.15 & 2.51 & 285 & 95 & 380 \\
\hline
\end{tabular}

Table 2. Shows sampling procedures participants selection in health institution of GamoGofa zone, 2016.

\begin{tabular}{|c|c|c|c|c|c|c|c|}
\hline & \multirow{2}{*}{ Name of health institution } & \multirow{2}{*}{ Pre-ART } & \multirow{2}{*}{ ART } & \multirow{2}{*}{ Total } & \multicolumn{2}{|c|}{ Required sample size } & \multirow{2}{*}{$\begin{array}{l}\text { Total } \\
\text { selected }\end{array}$} \\
\hline & & & & & (A) & (B) & \\
\hline 1 & Arba Minch Hospital & 1352 & 2415 & 3767 & 166 & 290 & 489 \\
\hline 2 & Chencha Hospital & 340 & 771 & 1111 & 40 & 88 & 128 \\
\hline 3 & Arba Minch HC & 242 & 677 & 919 & 28 & 76 & 104 \\
\hline 4 & Birbir HC & 104 & 207 & 311 & 12 & 24 & 36 \\
\hline 6 & Sawula Hospital & 140 & 280 & 420 & 16 & 32 & 48 \\
\hline \multirow[t]{2}{*}{7} & Sawula HC & 259 & 632 & 891 & 30 & 72 & 102 \\
\hline & Total & 2503 & 5109 & 7612 & 292 & 582 & 874 \\
\hline
\end{tabular}


on selected socio-demographic characteristics, lifestyle and behavioural characteristics including, physical activity, physical Measurements of weight, height, waist and hip circumference, and Blood glucose, as well as blood pressure.

Weight and height were measured with participants standing without shoes and wearing light clothing. Participants stood upright with the head in Frankfort plane for height measurement. Height was recorded to the nearest $0.5 \mathrm{~cm}$, and weight was recorded to the nearest $100 \mathrm{~g}$. Body-mass index (BMI) was calculated as weight in kilograms over height in meters squared [weight $(\mathrm{kg}) /($ height $\left.(\mathrm{m}))^{2}\right]$.

The global physical activity questionnaire (GPAQ) section of the STEPS instrument was used for assessment of physical activity, and total physical activity was presented in MET (metabolic equivalent) minutes per week. The instrument looks into three major domains of day-to-day activities; work (including domestic work), transport, and recreational activities. Level of total physical activity was subsequently classified into high, moderate, or low using the GPAQ analysis guideline provided along with the STEPS instrument. Blood pressure was measured in a sitting position using a digital device (Omron $M 4-I^{\circledR}$ ) after the study participant rested for at least five minutes. Three consecutive measurements were made in an interval of at least 3 minutes. Self-reported health status was determined by asking the participant.

\subsection{Data Quality Control}

Pre-test was conducted to identify a potential problem that was aroused during the actual data collection period. Trained ART nurses were used to collect the data after thoroughly explaining the objective of the study to each study subject and informed consent was obtained. There were also regular supervision and timely check-up of the completeness and consistency of responses to questions throughout the data collection period.

\subsection{Operational Definition}

Exposed: HIV patients who enrolled for chronic care and started HAART.

None exposed: HIV patients who enrolled for chronic care and not yet started HAART.

Outcome: an individual with sustained high blood pressure (SBP $\geq 140$ or DBP $\geq 90 \mathrm{mmHg}$ ) [18] or reported regular use of anti-hypertensive medication after ART follow up (s).

Hypertension: was defined as a sustained high blood pressure (SBP $\geq 140$ or DBP $\geq 90 \mathrm{mmHg}$ ) [18] or reported regular use of anti-hypertensive medication after ART follow up (s).

Body mass index (BMI): a measure of overall adiposity, will be categorized according to WHO criteria (underweight: BMI $<18.5 \mathrm{~kg} / \mathrm{m}^{2}$; normal: 18.5 - 24.9 $\mathrm{kg} / \mathrm{m}^{2}$; overweight: $25.0-29.9 \mathrm{~kg} / \mathrm{m}^{2}$; and obese: BMI $\left.\geq 30.0 \mathrm{~kg} / \mathrm{m}^{2}\right)$.

Alcohol consumption: it will be classified as low $(<1$ alcoholic beverage a 
week), moderate (1 - 21 alcoholic beverages a week), and high to excessive consumption (>21 alcoholic beverages a week) according to WHO classification.

\subsection{Data Processing and Analysis}

The data was cleaned to check for its completeness, consistency and the presence of missed values and variables. Any error identified was corrected as necessary. Then, it was entered into a pre-designed format in Epi-Info version 7 and transferred to SPSS version 21 for analysis. An assumption to apply binary logistic regression including fitness of model was assessed. Descriptive statistics were done using frequencies, percentages, mean, median, standard deviation and other summary statistics.

Binary Logistic regression Model was fitted first by Bivariable analysis between each independent variable and the dependent one. Independent variables with a p-value $<0.2$ was then included in the multivariate analysis. Those predictors with $\mathrm{p}$-value $<0.05$, in the multivariate analysis, was considered as independent and significant predictors for incidences of hypertension and was included in the final model. Crude and adjusted odds ratio with 95\% confidence interval was also be reported.

\subsection{Ethical Consideration}

Ethical clearance was obtained from Institutional Review office of Arba Minch University, college of medicine and Health Science. Permission letter was also obtained from Gamo-Gofa zone health departments and the departments also let respective hospitals and health centers to provide favorable media to conduct the study. Anonymity was maintained during abstraction of the data from the charts and informed verbal consents were taken from the respondents. Patients with hypertension and DM were informed about the status and refer to chronic hypertension and DM care follow up.

\section{Result and Discussion}

\subsection{Socio-Demographic and Economic Characteristics}

A total of $834 \mathrm{HIV} / \mathrm{AIDS}$ infected (95.4\% response rate) were included in this study. The mean age was $35.4 \pm 8.15$ years. More than half of them were Orthodox Christians (64.7\%) and married (56.7\%). More than three fourth (83.3\%) of them were from urban area and twenty three point four percent (195) were Housewife and about one third (42.0\%) were Gamo ethnicity (Table 3).

\subsection{Behavioral and Dietary Related Characteristics}

Fifty one participants (6.1\%) declared that they were smoking cigarettes previously. Concerning their alcohol use, 161 (19.3\%) were ever users. Eight seven (10.1\%) was involved in vigorous activities such as carrying or lifting heavy loads, and construction works. Most participants (97.4\%) use to walk for at least 10 minutes continuously every day. 
Table 3. Socio-demographic and Socioeconomic Characteristics of HIV infected Patients, at GGZ $(\mathrm{n}=834), 2016$.

\begin{tabular}{|c|c|c|c|}
\hline Variables & Responses & Frequency & $\%$ \\
\hline \multirow[t]{2}{*}{ Residence } & Urban & 695 & 83.3 \\
\hline & Rural & 139 & 16.7 \\
\hline \multirow[t]{2}{*}{ Sex } & Female & 471 & 56.5 \\
\hline & Male & 363 & 43.5 \\
\hline \multirow[t]{6}{*}{ Age } & $15-24$ & 31 & 3.7 \\
\hline & $25-34$ & 348 & 41.7 \\
\hline & $35-44$ & 319 & 38.2 \\
\hline & $45-54$ & 103 & 12.4 \\
\hline & $55-64$ & 16 & 1.9 \\
\hline & $65+$ & 4 & 0.5 \\
\hline \multirow[t]{4}{*}{ Educational Status } & No education & 194 & 23.3 \\
\hline & Primary education & 381 & 45.7 \\
\hline & Secondary education & 197 & 23.6 \\
\hline & Tertiary education & 62 & 7.4 \\
\hline \multirow[t]{5}{*}{ Marital status } & Married & 473 & 56.7 \\
\hline & Divorced & 96 & 11.5 \\
\hline & Single & 67 & 8.0 \\
\hline & Separated & 99 & 11.9 \\
\hline & Widowed & 99 & 11.9 \\
\hline \multirow[t]{6}{*}{ Occupational Status } & Housewife & 195 & 23.4 \\
\hline & Government employed & 187 & 22.4 \\
\hline & Merchant & 179 & 21.5 \\
\hline & Farmer & 147 & 17.6 \\
\hline & Un-employed & 54 & 6.5 \\
\hline & Others (student and self-employed) & 72 & 8.6 \\
\hline \multirow[t]{4}{*}{ Religion } & Orthodox & 540 & 64.7 \\
\hline & Protestant & 241 & 28.9 \\
\hline & Muslim & 36 & 4.3 \\
\hline & Others (Catholic and pagan) & 17 & 2.0 \\
\hline \multirow[t]{6}{*}{ Ethnicity } & Gamo & 350 & 42.0 \\
\hline & Gofa & 159 & 19.1 \\
\hline & Amhara & 125 & 15.0 \\
\hline & Wolaita & 94 & 11.3 \\
\hline & Konso & 11 & 1.3 \\
\hline & Others & 95 & 11.4 \\
\hline \multirow[t]{5}{*}{ Monthly income } & $\leq 750$ & 323 & 38.7 \\
\hline & $751-1300$ & 95 & 11.4 \\
\hline & $1301-2000$ & 145 & 17.4 \\
\hline & $>2000$ & 171 & 20.5 \\
\hline & Unknown & 100 & 12.0 \\
\hline
\end{tabular}


More than three fourth (83.9\%) of respondents reported that they usually use vegetables at least $1-3$ days in a week. Forty eight of the respondents (5.8\%) do not eat fruits at all in any days of a week. Only five of $(0.5 \%)$ respondents have reported use of hard drug like hashish and cocaine.

\subsection{Clinical Characteristics, HIV Infection and Antiretroviral Therapy}

Five hundred fifty (65.9\%) of PLHIV patient enrolled for HAART treatment and twenty one point one percent (176) were on 1a (30). Two percent (17) of PLHIV were known diabetic patient and more than three fourth $(84.5 \%)$ of the patients have no history of hypertensions. Nearly half of $(47.7 \%)$ of respondents has been started ART at WHO clinical stage and one third of the started at CD4 count of less than 200 (Table 4).

\subsection{Incidence of Systemic Hypertension}

The mean systolic and diastolic BP results were $115.7 \mathrm{mmHg}( \pm 16.1 \mathrm{SD})$ and 74.9 $\mathrm{mmHg}( \pm 12.4 \mathrm{SD})$. The overall incidence of hypertension was 20\% (95\% CI: 19.9 - 20.03), in equal proportion in Pre-HAART (20.1\%) and HAART (20.0\%) receiving patients (Figure 1).

\subsection{Bivariable Analysis}

In bivariable analysis, age, sex, residence and marital status were not significantly associated with Hypertension, but all other variables like modifiable (educational status, religion, occupation, and monthly income) and non-modifiable (ethnicity) and were significantly associated with incidence of Hypertension in cohort of HIV infected patients. In Bivariable analysis, only vigorous activities were significant and all other variables, like Tobacco use, fruit consumption and Duration on ART were not significant (Table 5 and Table 6).

\subsection{Predictors of Systemic Hypertension}

The study revealed modifiable; monthly income, Occupational status, marital status, Body mass index, and vigorous activities and non-modifiable Family history of hypertension were important predictors of hypertension among HIV infected patients. Other variables like educational status, religion and walking 10 minute per day were factors associated with Hypertension with consider ding the effect of other variables in multiple regression analysis, and it effect diluted in final analysis (Table 7).

\section{Discussion}

Hypertension as a well-known risk factor for CVDs \& it's disease of public health importance which occurred in a high incidence among HIV patients. In this study the prevalence of hypertension was $20 \%$ (95\% CI: 19.9 - 20.03), which was higher than the prevalence of hypertension in the Sub Saharan Africa 
Table 4. Clinical characteristics, HIV infection and antiretroviral therapy in cohort of HIV/AIDS Infected individuals in Gamo-Gofa Zone ( $\mathrm{n}=834), 2016$.

\begin{tabular}{|c|c|c|c|}
\hline Variables & Responses & Frequency & $\%$ \\
\hline \multirow[t]{2}{*}{ Base line HAART usage } & PRE-HAART & 284 & 34.1 \\
\hline & HAART & 550 & 65.9 \\
\hline \multirow[t]{7}{*}{ Type of drug regimen } & la (30) & 176 & 21.1 \\
\hline & 1a (40) & 3 & 0.4 \\
\hline & $1 \mathrm{~b}(30)$ & 128 & 15.3 \\
\hline & $1 \mathrm{~b}(40)$ & 3 & 0.4 \\
\hline & $1 c$ & 60 & 7.2 \\
\hline & $1 \mathrm{e}$ & 74 & 8.9 \\
\hline & $1 \mathrm{f}$ & 106 & 12.7 \\
\hline \multirow[t]{3}{*}{ History of Diabetes status } & Yes & 17 & 2.0 \\
\hline & No & 540 & 64.7 \\
\hline & Not known & 277 & 33.2 \\
\hline \multirow[t]{2}{*}{ Incidence of Hypertension } & Yes & 29 & 3.94 \\
\hline & No & 736 & 95.6 \\
\hline \multirow[t]{2}{*}{ Family History of hypertension } & Yes & 129 & 15.5 \\
\hline & No & 705 & 84.5 \\
\hline \multirow[t]{3}{*}{ History of UTI } & Yes & 76 & 9.1 \\
\hline & No & 755 & 90.5 \\
\hline & Unknown & 3 & 0.4 \\
\hline \multirow[t]{4}{*}{ WHO Clinical Stage } & Stage I & 290 & 34.8 \\
\hline & Stage II & 93 & 11.2 \\
\hline & Stage III & 398 & 47.7 \\
\hline & Stage IV & 53 & 6.4 \\
\hline \multirow[t]{3}{*}{ Base line CD4 lymphocyte Count } & $500+$ & 267 & 32.0 \\
\hline & $200-500$ & 294 & 35.3 \\
\hline & $<200$ & 273 & 32.7 \\
\hline \multirow[t]{3}{*}{ Duration of HAART Exposure } & Up to 1 year & & \\
\hline & 1 to 5 years & & \\
\hline & $5+$ Years & & \\
\hline \multirow[t]{3}{*}{$\operatorname{BMI}\left(\mathrm{kg} / \mathrm{m}^{2}\right)$} & $<18$ & 171 & 20.5 \\
\hline & $18-24$ & 544 & 65.2 \\
\hline & $25+$ & 119 & 14.3 \\
\hline
\end{tabular}

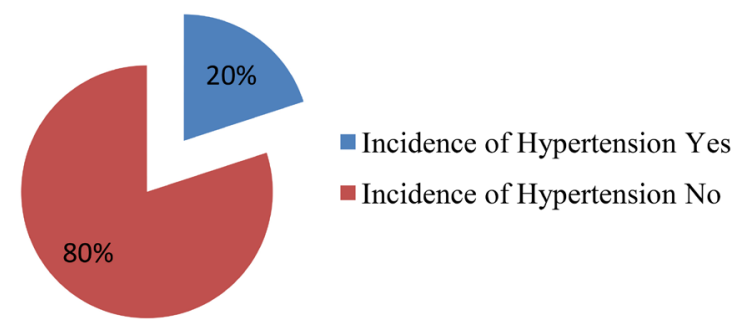

Figure 1. Incidence of systemic Hypertension among HIV infected patients in GGZ, 2016. 
Table 5. Bivariable regression analysis of factors associated with hypertension among study participants in Gamo-Gofa Zone $(\mathrm{n}=834), 2016$.

\begin{tabular}{|c|c|c|c|c|}
\hline \multirow{2}{*}{\multicolumn{2}{|c|}{ Variables }} & \multicolumn{2}{|c|}{ Incidence of HPN } & \multirow{2}{*}{$\begin{array}{c}\text { Odd Ratio } \\
\text { COR }(95 \% \mathrm{CI})\end{array}$} \\
\hline & & Yes (\%) & No (\%) & \\
\hline \multirow[t]{2}{*}{ Residence } & Urban & 137 & 558 & $0.89(0.57,1.39)$ \\
\hline & Rural & 30 & 109 & 1 \\
\hline \multirow[t]{2}{*}{ Sex } & Female & $92(19.5)$ & $379(80.5)$ & 1 \\
\hline & Male & $75(20.5)$ & $288(79.3)$ & $1.07(0.76,1.51)$ \\
\hline \multirow[t]{5}{*}{ Age } & $15-24$ & $2(6.5)$ & $29(93.5)$ & 1 \\
\hline & $25-34$ & $63(18.1)$ & $285(81.9)$ & $3.21(0.75,13.8)$ \\
\hline & $35-44$ & $71(22.3)$ & $248(77.7)$ & $4.15(0.97,17.8)$ \\
\hline & $45-54$ & $24(23.3)$ & $79(76.7)$ & $4.41(0.98,19.8)$ \\
\hline & $55-64$ & $3(15.0)$ & $17(85.0)$ & $2.56(0.39,16.8)$ \\
\hline \multirow{4}{*}{$\begin{array}{l}\text { Educational } \\
\text { Status }\end{array}$} & No education & $27(13.9)$ & $167(86.1)$ & 1 \\
\hline & Primary education & $60(15.7)$ & $321(84.3)$ & $1.16(0.71,1.89)$ \\
\hline & Secondary education & $56(28.4)$ & $141(71.6)$ & $2.46(1.47,4.09)$ \\
\hline & Tertiary education & $24(38.7)$ & $38(61.3)$ & $3.91(2.03,7.51)$ \\
\hline \multirow{6}{*}{$\begin{array}{c}\text { Occupational } \\
\text { Status }\end{array}$} & Housewife & $43(22.1)$ & $152(77.9)$ & 1 \\
\hline & Government employed & $61(32.6)$ & $126(67.4)$ & $1.71(1.08,2.70)$ \\
\hline & Merchant & $15(8.4)$ & $164(91.6)$ & $0.32(0.17,0.61)$ \\
\hline & Farmer & $35(23.8)$ & $112(76.2)$ & $1.10(0.66,1.83)$ \\
\hline & Un-employed & $8(14.8)$ & $46(85.2)$ & $0.62(0.27,1.40)$ \\
\hline & Others (student and self-employed) & $5(6.9)$ & $67(93.1)$ & $0.26(0.10,0.69)$ \\
\hline \multirow[t]{4}{*}{ Religion } & Orthodox & $119(22.0)$ & $421(78.0)$ & 1 \\
\hline & Protestant & $35(14.5)$ & $206(85.5)$ & $0.60(0.39,0.91)$ \\
\hline & Muslim & $13(36.1)$ & $23(63.9)$ & $2.00(0.98,4.07)$ \\
\hline & Others (Catholic and pagan) & $0(0.0)$ & $17(100.0)$ & - \\
\hline \multirow[t]{5}{*}{ Monthly income } & $\leq 750$ & $39(12.1)$ & $284(87.9)$ & 1 \\
\hline & $751-1300$ & $15(15.8)$ & $80(84.2)$ & $1.36(0.72,2.60)$ \\
\hline & $1301-2000$ & $49(33.8)$ & $96(66.2)$ & $3.71(2.30,6.01)$ \\
\hline & $>2000$ & $57(33.3)$ & $114(66.7)$ & $3.64(2.29,5.78)$ \\
\hline & Unknown & & & \\
\hline
\end{tabular}

Table 6. Bivariable regression analysis of factors associated with hypertension among study participants in Gamo-Gofa Zone ( $\mathrm{n}=834), 2016$.

\begin{tabular}{ccccc}
\hline \multirow{2}{*}{ Variables } & \multicolumn{2}{c}{ Incidence of HPN } & \multicolumn{2}{c}{ Odd Ratio } \\
\cline { 2 - 5 } & Yes (\%) & No (\%) & COR (95\% CI) & AOR (95\% CI) \\
\hline \multirow{2}{*}{ Alcohol use } & No & $132(19.6)$ & $541(80.4)$ & 1 \\
& Yes & $35(21.9)$ & $125(78.1)$ & $1.15(0.75,1.75)$ \\
Fruit consumption/week & None & $12(25.0)$ & $36(75.0)$ & $1.69(0.71,4.03)$ \\
& $1-3$ days & $136(20.3)$ & $533(79.7)$ & $1.29(0.71,2.37)$ \\
& $4-7$ days & $14(16.5)$ & $71(83.5)$ & 1 \\
\hline
\end{tabular}




\section{Continued}

\begin{tabular}{|c|c|c|c|c|}
\hline \multirow[t]{3}{*}{ Vegetable use/week } & None & $0(0.0)$ & $8(100.0)$ & - \\
\hline & $1-3$ days & $142(20.3)$ & $558(79.7)$ & $1.03(0.59,1.78)$ \\
\hline & 4 - 7 days & $18(19.8)$ & $73(80.2)$ & 1 \\
\hline \multirow[t]{2}{*}{ Vigorous activities/week } & None & $165(22.1)$ & $582(77.9)$ & $12.0(2.93,48.5)$ \\
\hline & $1-4$ & $2(2.3)$ & $85(97.7)$ & 1 \\
\hline \multirow[t]{4}{*}{ Walking status } & None & $160(19.7)$ & $652(80.3)$ & 1 \\
\hline & $1-3$ & $4(40.0)$ & $6(60.0)$ & $2.71(0.76,0.97)$ \\
\hline & $4-7$ & $2(20.0)$ & $8(80.0)$ & $1.02(0.21,4.84)$ \\
\hline & Daily & $1(50.0)$ & $1(50.0)$ & $4.07(0.25,65.5)$ \\
\hline \multirow[t]{3}{*}{ BMI $\left(\mathrm{kg} / \mathrm{m}^{2}\right)$} & $<18$ & $8(4.7)$ & $163(95.3)$ & 1 \\
\hline & $18-24$ & $104(19.1)$ & $440(80.9)$ & $4.82(2.29,10.1)$ \\
\hline & $25+$ & $55(46.2)$ & $64(53.8)$ & $17.5(7.89,38.8)$ \\
\hline \multirow[t]{2}{*}{ Base line HAART usage } & Yes & $57(20.1)$ & $226(79.9)$ & $1.01(0.70,1.45)$ \\
\hline & No & $110(20.0)$ & $41(80.0)$ & 1 \\
\hline \multirow[t]{8}{*}{ Type of drug regimen } & No & $57(20.1)$ & $227(79.9)$ & 1 \\
\hline & 1a (30) & $24(13.6)$ & $152(86.4)$ & $0.63(0.37,1.06)$ \\
\hline & 1a (40) & $1(33.3)$ & $2(66.7)$ & $1.99(0.18,22.4)$ \\
\hline & $1 \mathrm{~b}(30)$ & $29(22.7)$ & $99(77.3)$ & $1.16(0.70,1.93)$ \\
\hline & $1 \mathrm{~b}(40)$ & $0(0.0)$ & $3(100.0)$ & - \\
\hline & $1 \mathrm{c}$ & $13(21.7)$ & $47(78.3)$ & $1.10(0.59,2.17)$ \\
\hline & le & $14(18.9)$ & $60(81.1)$ & $0.93(0.48,1.78)$ \\
\hline & $1 \mathrm{f}$ & $29(27.4)$ & $77(72.6)$ & $1.50(0.89,2.51)$ \\
\hline \multirow[t]{2}{*}{ Diabetes status } & Yes & $112(20.7)$ & $428(79.3)$ & $1.96(0.42,8.70)$ \\
\hline & No & $2(11.8)$ & $15(88.2)$ & 1 \\
\hline \multirow{3}{*}{$\begin{array}{c}\text { Base line CD4 lymphocyte } \\
\text { Count }\end{array}$} & $500+$ & $53(19.4)$ & $220(80.6)$ & $0.97(0.64,1.49)$ \\
\hline & $200-500$ & $61(20.7)$ & $233(79.3)$ & $1.06(0.70,1.59)$ \\
\hline & $<200$ & $53(19.9)$ & $214(80.1)$ & 1 \\
\hline \multirow{2}{*}{$\begin{array}{l}\text { Family History of Diabetes } \\
\text { status }\end{array}$} & No & $149(19.3)$ & $624(80.7)$ & 1 \\
\hline & Yes & $18(29.5)$ & $43(70.5)$ & $1.75(0.98,3.13)$ \\
\hline \multirow{2}{*}{$\begin{array}{l}\text { Family History of } \\
\text { hypertension }\end{array}$} & No & $124(17.6)$ & $581(82.4)$ & 1 \\
\hline & Yes & $43(33.3)$ & $86(66.7)$ & $2.34(1.55,3.55)$ \\
\hline \multirow[t]{3}{*}{ History of UTI } & Yes & $26(34.2)$ & $50(65.8)$ & \\
\hline & No & $138(18.3)$ & $617(81.7)$ & \\
\hline & Unknown & $3(100.0)$ & $0(0.0)$ & \\
\hline \multirow[t]{4}{*}{ WHO Clinical Stage } & Stage I & $55(19.0)$ & $235(81.0)$ & 1 \\
\hline & Stage II & $18(19.4)$ & $75(80.0)$ & $1.03(0.57,1.85)$ \\
\hline & Stage III & $87(21.9)$ & $311(78.1)$ & $1.19(0.82,1.74)$ \\
\hline & Stage IV & $7(13.2)$ & $46(86.8)$ & $0.65(0.28,1.52)$ \\
\hline \multirow{3}{*}{$\begin{array}{c}\text { Base line CD4 lymphocyte } \\
\text { Count }\end{array}$} & $500+$ & $53(19.4)$ & $220(80.6)$ & 1 \\
\hline & $200-500$ & $61(20.7)$ & $233(79.3)$ & $0.97(0.64,1.49)$ \\
\hline & $<200$ & $53(19.9)$ & $214(80.1)$ & $1.06(0.70,1.59)$ \\
\hline
\end{tabular}


Table 7. Predictors associated with Incidence of Hypertension among Cohort of HIV patients in GGZ, 2016.

\begin{tabular}{|c|c|c|c|c|c|}
\hline \multirow{2}{*}{ Variables } & \multirow{2}{*}{ Responses } & \multicolumn{2}{|c|}{ Incidence of HPN } & \multicolumn{2}{|c|}{ Odd Ratio } \\
\hline & & Yes (\%) & No (\%) & COR $(95 \% \mathrm{CI})$ & $\operatorname{AOR}(95 \% \mathrm{CI})$ \\
\hline \multirow[t]{5}{*}{ Age } & $15-24$ & $2(6.5)$ & $29(93.5)$ & 1 & - \\
\hline & $25-34$ & $63(18.1)$ & $285(81.9)$ & $3.21(0.75,13.8)$ & \\
\hline & $35-44$ & $71(22.3)$ & $248(77.7)$ & $4.15(0.97,17.8)$ & \\
\hline & $45-54$ & $24(23.3)$ & $79(76.7)$ & $4.41(0.98,19.8)$ & \\
\hline & $55-64$ & $3(15.0)$ & $17(85.0)$ & $2.56(0.39,16.8)$ & \\
\hline \multirow[t]{4}{*}{ Educational Status } & No education & $27(13.9)$ & $167(86.1)$ & 1 & - \\
\hline & Primary education & $60(15.7)$ & $321(84.3)$ & $1.16(0.71,1.89)$ & \\
\hline & Secondary education & $56(28.4)$ & $141(71.6)$ & $2.46(1.47,4.09)$ & \\
\hline & Tertiary education & $24(38.7)$ & $38(61.3)$ & $3.91(2.03,7.51)$ & \\
\hline \multirow[t]{6}{*}{ Occupational Status } & Housewife & $43(22.1)$ & $152(77.9)$ & 1 & 1 \\
\hline & Government employed & $61(32.6)$ & $126(67.4)$ & $1.71(1.08,2.70)$ & $1.18(0.65,2.12)$ \\
\hline & Merchant & $15(8.4)$ & $164(91.6)$ & $0.32(0.17,0.61)$ & $0.36(0.17,0.78)$ \\
\hline & Farmer & $35(23.8)$ & $112(76.2)$ & $1.10(0.66,1.83)$ & $0.77(0.41,1.44)$ \\
\hline & Un-employed & $8(14.8)$ & $46(85.2)$ & $0.62(0.27,1.40)$ & $0.51(0.20,1.27)$ \\
\hline & Others (student and self-employed) & $5(6.9)$ & $67(93.1)$ & $0.26(0.10,0.69)$ & $0.7(0.15,3.37)$ \\
\hline \multirow[t]{4}{*}{ Religion } & Orthodox & $119(22.0)$ & $421(78.0)$ & 1 & 1 \\
\hline & Protestant & $35(14.5)$ & $206(85.5)$ & $0.60(0.39,0.91)$ & $0.75(0.47,1.20)$ \\
\hline & Muslim & $13(36.1)$ & $23(63.9)$ & $2.00(0.98,4.07)$ & $1.71(0.74,3.91)$ \\
\hline & Others (Catholic and pagan) & $0(0.0)$ & $17(100.0)$ & - & - \\
\hline \multirow[t]{6}{*}{ Ethnicity } & Amhara & $33(26.4)$ & $92(73.6)$ & 1 & \\
\hline & Gamo & $68(19.4)$ & $282(80.6)$ & $0.67(0.42,1.08)$ & - \\
\hline & Gofa & $24(15.1)$ & $135(84.9)$ & $0.49(0.28,0.89)$ & \\
\hline & Wolaita & $3(27.3)$ & $8(72.7)$ & $1.05(0.26,4.18)$ & \\
\hline & Konso & $28(29.5)$ & $67(70.5)$ & $1.17(0.64,2.11)$ & \\
\hline & Others & & & $0.36(0.18,0.78)$ & \\
\hline \multirow[t]{4}{*}{ Monthly income } & $\leq 750$ & $39(12.1)$ & $284(87.9)$ & 1 & 1 \\
\hline & $751-1300$ & $15(15.8)$ & $80(84.2)$ & $1.36(0.72,2.60)$ & $0.88(0.42,1.84)$ \\
\hline & $1301-2000$ & $49(33.8)$ & $96(66.2)$ & $3.71(2.30,6.01)$ & $2.26(1.26,4.05)$ \\
\hline & $>2000$ & $57(33.3)$ & $114(66.7)$ & $3.64(2.29,5.78)$ & $1.94(.04,3.62)$ \\
\hline \multirow[t]{3}{*}{ Fruit consumption/week } & None & $12(25.0)$ & $36(75.0)$ & $1.69(0.71,4.03)$ & - \\
\hline & 1 - 3 days & $136(20.3)$ & $533(79.7)$ & $1.29(0.71,2.37)$ & \\
\hline & 4 - 7 days & $14(16.5)$ & $71(83.5)$ & 1 & \\
\hline \multirow[t]{2}{*}{ Vigorous Activities/week } & None & $165(22.1)$ & $582(77.9)$ & $12.0(2.93,48.5)$ & $4.91(1.12,21.6)$ \\
\hline & $1-4$ & $2(2.3)$ & $85(97.7)$ & 1 & 1 \\
\hline \multirow[t]{4}{*}{ Walking status } & None & $160(19.7)$ & $652(80.3)$ & 1 & \\
\hline & $1-3$ & $4(40.0)$ & $6(60.0)$ & $2.71(0.76,0.97)$ & - \\
\hline & $4-7$ & $2(20.0)$ & $8(80.0)$ & $1.02(0.21,4.84)$ & \\
\hline & Daily & $1(50.0)$ & $1(50.0)$ & $4.07(0.25,65.5)$ & \\
\hline
\end{tabular}


Continued

\begin{tabular}{|c|c|c|c|c|c|}
\hline \multirow[t]{3}{*}{ BMI $\left(\mathrm{kg} / \mathrm{m}^{2}\right)$} & $<18$ & $8(4.7)$ & $163(95.3)$ & 1 & 1 \\
\hline & $18-24$ & $104(19.1)$ & $440(80.9)$ & $4.82(2.29,10.1)$ & $4.24(1.93,9.30)$ \\
\hline & $25+$ & $55(46.2)$ & $64(53.8)$ & $17.5(7.89,38.8)$ & $12.6(5.32,29.8)$ \\
\hline \multirow[t]{8}{*}{ Type of drug regimen } & No & $57(20.1)$ & $227(79.9)$ & 1 & - \\
\hline & 1a (30) & $24(13.6)$ & $152(86.4)$ & $0.63(0.37,1.06)$ & \\
\hline & 1a $(40)$ & $1(33.3)$ & $2(66.7)$ & $1.99(0.18,22.4)$ & \\
\hline & $1 \mathrm{~b}(30)$ & $29(22.7)$ & $99(77.3)$ & $1.16(0.70,1.93)$ & \\
\hline & $1 \mathrm{~b}(40)$ & $0(0.0)$ & $3(100.0)$ & - & \\
\hline & $1 \mathrm{c}$ & $13(21.7)$ & $47(78.3)$ & $1.10(0.59,2.17)$ & \\
\hline & $1 \mathrm{e}$ & $14(18.9)$ & $60(81.1)$ & $0.93(0.48,1.78)$ & \\
\hline & 1f & $29(27.4)$ & $77(72.6)$ & $1.50(0.89,2.51)$ & \\
\hline \multirow[t]{2}{*}{ Family History of DM } & No & $149(19.3)$ & $624(80.7)$ & 1 & - \\
\hline & Yes & $18(29.5)$ & $43(70.5)$ & $1.75(0.98,3.13)$ & \\
\hline \multirow{2}{*}{$\begin{array}{l}\text { Family History of hyper- } \\
\text { tension }\end{array}$} & No & $124(17.6)$ & $581(82.4)$ & 1 & 1 \\
\hline & Yes & $43(33.3)$ & $86(66.7)$ & $2.34(1.55,3.55)$ & $2.18(1.32,3.58)$ \\
\hline
\end{tabular}

(16.2\%), and lower than study done in Uganda (27.2\%), in Europe (44.4\%), and study done in India (44.8\%) [19] [20] [21] [22]. Compared to current findings the higher prevalence of hypertension was observed in the study done among HIV naïve individuals in Addis Ababa (27.3\%), North West Ethiopia (28.5\%) and study done in south-western Ethiopia (3.2\%) [23] [24] [25].

One possible reason is due to the difference in cut-off points for definition of hypertension between the current study which used the NCEP guidelines which considered a lower cut-off point level BP $>140 / 90 \mathrm{mmHg}$, than NHMS Survey which defined hypertension as $(\mathrm{BP}>130 / 85 \mathrm{mmHg}$ ). Other studies also reported a lower prevalence of hypertension compare to our study due to the higher level of definition for blood pressure to be termed as hypertensive. Generally, differences in the study population characteristics, de-sign and methodology, the type and number of variables taken as risk factors, cut-off points and references for definition of hypertension cause a variation in incidence of hypertension.

Growing body of epidemiologic evidence suggests that there are a number of modifiable and non-modifiable factors that lead to increased risk of hypertension in low income country like Ethiopia, in thus study modifiable characteristics; like monthly income, Occupational status, Body mass index, and vigorous activities) and non-modifiable character like, Family history of hypertension, were important predictors of Incidence of hypertension among HIV infected patients.

Among modifiable factors; occupation status was significantly associated with incidence of hypertension among HIV infected patients. Accordingly; HIV patient of merchant occupation was $64 \%$ (AOR, 0.36 ; $95 \%$ CI; $(0.17,0.78)$ ) less risk of developing Hypertension than Housewife.

Monthly income was other modifiable predictors of Hypertension in HIV pa- 
tients, according to this study, an individual whose income were (1301 - 2000) and greater than 2000 were 2.3 (AOR; 2.26, 95\% CI $(1.26,4.05)$ ) and 2 times (AOR, 1.94, 95\% $(0.04,3.62)$ ) higher risk of developing Hypertension than HIV infected patients whose income is less than 750 birr per months. This might related to eating or dietary habit, which in turn related with economic status, thus an individual who earn more money may utilize fatty food which increase the atherosclerosis which may in turn block Blood vessels.

In the current study, obese HIV patients had a 13 times (AOR: 12.6, (95\% CI: $5.32,29.8)$ ) higher risk of developing systemic hypertension compared to those with underweight patients. Which was in-line with the studies done in India and case control study in Brazil [26] [27] [28] [29], Consistent result also was observed in the study done in south western part of Ethiopia [25].

Family history of hypertension was significantly associated with being hypertensive in this study. In current study, HIV patients with family history of hypertension were 2.1 times (AOR: $2.18,95 \%$ CI: 1.32, 3.58) higher risk of developing Hypertension than without having family history of hypertension. It was consistent with study done in south western part of Ethiopia, which showed Family history of hypertension, as risk factors for HPN [25].

Other modifiable predictors of incidence of hypertension were vigorous activities, that HIV patients who would not involve in vigorous activities such as carrying or lifting heavy loads, and construction works were 5 times (AOR, 4.91, 95\% CI, $(1.12,21.6))$ higher risk of developing systemic hypertension than those who liked to be involved in vigorous activities.

Unlike other studies done so far [28] [30] cigarette smoking, use of alcohol and age were not significantly associated with hypertension in this study. This may be due to the low prevalence of these factors in the HIV/AIDS infected patients.

Modifiable (educational status, religion, occupation, and monthly income) and non-modifiable (ethnicity) and were significantly associated with incidence of Hypertension in Bivariable analysis and the significance is diluted in multiple logistic regression analysis.

This study has potential limitations. Firstly, it was difficult to know the baseline status of systemic hypertension in some charts and excluded which may bias the finding of current research.

Second, this study is limited to behavioral and physical measurements, and did not include biochemical measurements such as a 24 hours urine sodium concentration, cholesterols and, etc. Thirdly, the lower age included in this study was 17 which made comparisons with other studies difficult.

\section{Conclusion}

There was high incidence of hypertension among HIV infected patients in Health institution of Gamo Gofa zone and may reflect insights for intervention. The investigators accept the null hypothesis that there was association between 
HAART intervention and Incidence of Hypertension. Monthly income, occupational status, marital status, body mass index, vigorous activities and Family history of hypertension, were predictors of systemic hypertension among HIV/AIDS infected patients. Federal ministry of health should design and implement of early screening programs and prompt treatment for systemic hypertension among PLHIV. HIV patients should be advised to adopt a healthy lifestyle like avoiding sedentary life style and regular monitoring of blood pressure. Since the limitation of the study was difficult to know the baseline status of systemic hypertension in some charts and excluded which may bias the finding of current research, so that prospective study should be done to see real effect of HAART on systemic hypertension among PLHIIV patients.

\section{Acknowledgements}

I would like to thank Arba Minch University, research directorate office for giving us an initiative and fund to carry out this study. My gratitude also goes to staffs of health institution and Gamo Gofa Zone Health department for providing us the base line data. I would also extend our appreciation to supervisors and data collectors for their data collection, supervision and collection and unwavering support for the successful completion of research work. The last but not the least, our thanks goes to my family (My wife and Daughter) for letting me to write report, and as well as timely accomplishments of the duties given.

\section{Data Availability}

Upon acceptance of manuscript, we agree to make data available for readers based on journal guideline.

\section{Funding Statement}

Funding for this particular research was obtained from Arba Minch University, college of medicine and health science as part of staff research funding.

\section{Conflicts of Interest}

The author declares no conflicts of interest regarding the publication of this paper.

\section{References}

[1] Kotchen, T.A. (2007) Hypertension Control: Trends, Approaches, and Goals. Hypertension, 49, 19-20. https://doi.org/10.1161/01.HYP.0000250394.05703.06

[2] Chobanian, A.V., Bakris, G.L., Black, H.R., et al. (2003) National Heart, Lung, and Blood Institute Joint National Committee on Prevention, Detection, Evaluation, and Treatment of High Blood Pressure; National High Blood Pressure Education Program Coordinating Committee. The Seventh Report of the Joint National Committee on Prevention, Detection, Evaluation, and Treatment of High Blood Pressure: The JNC 7 Report. The Journal of the American Medical Association, 289, 2560-2572. https://doi.org/10.1001/jama.289.19.2560 
[3] World Health Organization (2002) The World Health Report 2002: Reducing Risks, Promoting Healthy Life. World Health Organization, Geneva, Switzerland.

[4] Hadigan, C., Meigs, J.B., Corcoran, C., et al. (2001) Metabolic Abnormalities and Cardiovascular Disease Risk Factors in Adults with Human Immunodeficiency Virus Infection and Lipod-Dystrophy. Clinical Infectious Diseases, 32, 130-139. https://doi.org/10.1086/317541

[5] Nicastri, E., et al. (2007) Sex Issues in HIV-1-Infected Persons during Highly Active Antiretroviral Therapy: A Systematic Review. Journal of Antimicrobial Chemotherapy, 60, 724-732. https://doi.org/10.1093/jac/dkm302

[6] Antiretroviral Therapy Cohort Collaboration (2008) Life Expectancy of Individuals on Combination Antiretroviral Therapy in High-Income Countries: A Collaborative Analysis of 14 Cohort Studies. The Lancet, 372, 293s-299s. https://doi.org/10.1016/S0140-6736(08)61113-7

[7] Baekken, M., et al. (2008) Hypertension in an Urban HIV-Positive Population Compared with the General Population: Influence of Combination Antiretroviral Therapy. Journal of Hypertension, 26, 2126-2133. https://doi.org/10.1097/HJH.0b013e32830ef5fb

[8] Friis-Møller, N., Reiss, P., Sabin, C.A., et al. (2007) Class of Antiretroviral Drugs and the Risk of Myocardial Infarction. The New England Journal of Medicine, 356, 1723-1735. https://doi.org/10.1056/NEJMoa062744

[9] Winston, J. and Klotman, P.E. (1998) HIV-Associated Nephropathy. Mount Sinai Journal of Medicine, 65, 27-32.

[10] Aoun, S. and Ramos, E. (2000) Hypertension in the HIV-Infected Patient. Current Hypertension Reports, 2, 478-481. https://doi.org/10.1007/s11906-000-0031-1

[11] Chow, D.C., Souza, S.A., Chen, R., et al. (2003) Elevated Blood Pressure in HIVInfected Individuals Receiving Highly Active Antiretroviral Therapy. HIV Clinical Trials, 4, 411-416. https://doi.org/10.1310/5E7Q-PGWB-16UE-J48U

[12] Coloma Conde, A.G., Alvarez Albarrán, M., Roca-Cusachs, C.A., et al. (2008) Prevalence of Arterial Hypertension and Lipid Profile in HIV Patients. Medical Clinics, 131, 681-684. https://doi.org/10.1157/13129111

[13] Dubé, M.P., Lipshultz, S.E., Fichenbaum, C.J., et al. (2008) Effects of HIV Infection and Antiretroviral Therapy on the Heart and Vasculature. Circulation, 118, e36-e40. https://doi.org/10.1161/CIRCULATIONAHA.107.189625

[14] Bergersen, B.M., Sandvik, L., Dunlop, O., et al. (2003) Prevalence of Hypertension in HIV-Positive Patients on Highly Active Retroviral Therapy (HAART) Compared with HAART-Naive and HIV-Negative Controls: Results from a Norwegian Study of 721 Patients. European Journal of Clinical Microbiology \& Infectious Diseases, 22, 731-736. https://doi.org/10.1007/s10096-003-1034-Z

[15] Thiébaut, R., El-Sadr, W.M., Friis-Møller, N. and Data Collection of Adverse Events of Anti-HIV Drugs Study Group (2005) Predictors of Hypertension and Changes of Blood Pressure in HIV-Infected Patients. Antiviral Therapy, 10, 811s-823s.

[16] Crane, H.M., Van Rompaey, S.E. and Kitahata, M.M. (2006) Antiretroviral Medications Associated with Elevated Blood Pressure among Patients Receiving Highly Active Antiretroviral Therapy. AIDS, 20, 1019-1026. https://doi.org/10.1097/01.aids.0000222074.45372.00

[17] Bonita, R., de Courten, M., Dwyer, T., Jamrozik, K. and Winkelmann, R. (2002) Surveillance of Risk Factors for Noncommunicable Diseases: The WHO STEP Wise Approach. World Health Organization, Geneva. http://www.who.int/chp/steps 
[18] Mathers, C., Stevens, G. and Mascarenhas, M. (2009) Global Health Risks: Mortality and Burden of Disease Attributable to Selected Major Risks. World Health Organization, Geneva, Switzerland.

[19] Twagirumukiza, M., De Bacquer, D., Kips, J.G., de Backer, G., Stichele, R.V. and Van Bortel, L.M. (2011) Current and Projected Prevalence of Arterial Hypertension in Sub-Saharan Africa by Sex, Age and Habitat: An Estimate from Population Studies. Journal of Hypertension, 29, 1243-1252.

https://doi.org/10.1097/HJH.0b013e328346995d

[20] Musinguzi, G. and Nuwaha, F. (2013) Prevalence, Awareness and Control of Hypertension in Uganda. PLoS ONE, 8, e62236.

https://doi.org/10.1371/journal.pone.0062236

[21] Wolf-Maier, K., Cooper, R.S., Banegas, J.R., et al. (2003) Hypertension Prevalence and Blood Pressure Levels in 6 European Countries, Canada, and the United States. Journal of the American Medical Association, 289, 2363-2369. https://doi.org/10.1001/jama.289.18.2363

[22] Chaturvedi, S., Pant, M., Yadav, G. and Neelam. (2007) Hypertension in Delhi: Prevalence, Awareness, Treatment and Control. Tropical Doctor, 37, 142-145. https://doi.org/10.1258/004947507781524593

[23] Angaw, K., Dadi, A.F. and Alene, K.A. (2015) Prevalence of Hypertension among Federal Ministry Civil Servants in Addis Ababa, Ethiopia: A Call for a WorkplaceScreening Program. BMC Cardiovascular Disorders, 15, 76. https://doi.org/10.1186/s12872-015-0062-9

[24] Awoke, A., et al. (2012) Prevalence and Associated Factors of Hypertension among Adults in Gondar, Northwest Ethiopia: A Community Based Cross-Sectional Study. BMC Cardiovascular Disorders, 12, 113. https://doi.org/10.1186/1471-2261-12-113

[25] Bissa, S., Mossie, A. and Gobena, T. (2012) Prevalence of Hypertension and Its Association with Substance Use among Adults Living in Jimma Town, South West Ethiopia. World Journal of Medicine and Medical Science, 2, 1-11.

[26] Agrawal, V.K., Bhalwar, R. and Basannar, D.R. (2008) Prevalence and Determinants of Hypertension in a Rural Community of India. Medical Journal Armed Forces India, 64, 21-25. https://doi.org/10.1016/S0377-1237(08)80139-6

[27] Agrawal, A., et al. (2015) Research Article A Study of Risk Factors and Impact of HAART on Blood Pressure in North Indians Living with HIV/AIDS. Scholars Academic Journal of Biosciences (SAJB), 3, 4.

[28] Midha, T., et al. (2009) Prevalence and Determinants of Hypertension in the Urban and Rural Population of a North Indian District. East African Journal of Public Health, 6, 268-273.

[29] de Arruda Junior, E.R., et al. (2010) Risk Factors Related to Hypertension among Patients in a Cohort Living with HIV/AIDS. Journal of Infectious Disease, 14, 281-287. https://doi.org/10.1590/S1413-86702010000300014

[30] Chow, D., et al. (2000) Epidemiologic Evidence of Increasing Blood Pressure in HIV-1 Infected Individuals in the Era of HAART. Antiviral Therapy, 5, S31. 